\title{
OVERVIEW OF OPTIMIZATION ALGORITHMS FOR UAVS POSITIONING
}

\author{
Adrián STOSIL*, Marcel VOLOŠIN**, Taras MAKSYMYUK**, Gabriel BUGÁR* \\ ${ }^{*}$ Department of Electronics and Multimedia Communications, Faculty of Electrical Engineering and Informatics, Technical \\ University of Košice, Němcovej 32, 04001 Košice, Slovak Republic, tel. +421 55602 2808, E-mail: gabriel.bugar@tuke.sk \\ ${ }^{* *}$ Department of Computers and Informatics, Faculty of Electrical Engineering and Informatics, \\ Technical University of Košice, Letná 9, 04200 Košice, Slovak Republic \\ ${ }^{* * *}$ Department of Telecommunications, Lviv Polytechnic National University, Ukraine
}

\begin{abstract}
Mobile broadband networks can provide a reliable and flexible communication channel. User requirements can come with different levels of specificity. The widespread application of unmanned aerial vehicles (UAVs, commonly known as a drones) introduces possibilities of use in modern upcoming mobile networks; for example, $5 G$ and $6 G$, to achieve and support various use cases from low latency to high bandwidth scenarios. For reliable command and control communication, mobile networks can provide flexible differentiated QoS matching the needed reliability, latency and throughput. Many end user equipment connected to the same base station can overload the system and may cause the network to be unavailable. Also, in case the fixed infrastructure is partially decommissioned, destroyed or the network is congested and the system capacity is not sufficient, it is appropriate to use autonomous drones as mobile base stations to ensure well signal coverage of the affected area. The aim of this work is to overview of optimization algorithm developed to provide the best drones' locations. We compared the techniques of minimization of the number of drones needed to cover users located in a given area with respect to the time required to calculate the optimal positions of the drones.
\end{abstract}

Keywords: autonomous drone, mobile base stations, fixed infrastructure, 5G, $6 G$

\section{INTRODUCTION}

From the beginning of mobile wireless communication in the late 1970s, from analogue voice calls to today's highquality mobile broadband services, a new generation supports a new cellular system every ten years. The first generation (1G) communication system dates from 1982, while $2 \mathrm{G}$ systems were commercially deployed in 1992 , followed by $3 \mathrm{G}$ systems, and since 2010 the $4 \mathrm{G}$ network has been in use. It is expected that in the near future the current $4 \mathrm{G}$ network will meet its capacity limits, and therefore it will be necessary to look for new solutions to this problem [1].

The upcoming fifth generation of mobile networks brings higher data rates, lower response, and increases that allows it to connect to more devices than current fourthgeneration networks. We need to realize that mobile networks have been with us slowly for 30 years. The first $2 \mathrm{G}$ network was launched in Finland, and while some have no idea what the $5 \mathrm{G}$ network is, $5 \mathrm{G}$ networks have already been launched in Switzerland, China or South Korea for useful purposes. But 5G networks are not as groundbreaking in the use of new technologies as they may seem at first glance. 5G networks largely require existing technologies that have been in place for several years [2]. There are many studies that show that $5 \mathrm{G}$ networks will no longer have an impact on human health. The frequencies that will also serve $5 \mathrm{G}$ networks are also used, for example, by DVB-T digital television. Although current television transmitters have outputs of tens to hundreds of $\mathrm{kW}$, they are located on distant hills above the cities and cover a large area. Operators will prepare them for the functionality of the $5 \mathrm{G}$ network, but will continue to produce lower performance. With increasing demands from operators or users for transmission speed, low latency, greater transmission reliability, large network capacity, increased availability cover or the number of devices that require connection security, the advancement of mobile networks is advancing [3]. 5G networks provide much greater possibilities than previous networks in previous years and new possibilities of use.

\section{RECENT NETWORK INFRASTRUCTURE}

The first real step towards GSM dates back to 1982, when a group of European telecommunications operators asked the CEPT for the possibility of standardizing a panEuropean mobile communications standard. This created a working group called Groupe Spécial Mobile (hence the acronym GSM, which later received a less French interpretation). The abbreviation GSM is still used for the system today, but instead of the interpretation of Groupe Spécial Mobile, the more apt Global System for Mobile Communications is used today [4]. The first concern of the GSM Group was to create a list of requirements that are placed on the new mobile system. Since then, the development of GSM networks has expanded and the individual generations from $1 \mathrm{G}$ to $5 \mathrm{G}$ are outlined in Fig 1 .

\begin{tabular}{|c|c|l|c|}
\hline 16 & 1980 & $\begin{array}{l}\text { - analog transmission (prone to interference) } \\
\text { - no support of encryption systems } \\
\text { - FDMA }\end{array}$ & 2,4 kbps \\
\hline 26 & 1990 & $\begin{array}{l}\text { - voice and digital communication } \\
\text { - circuits and packet switching technologies } \\
\text { - CDMA, TDMA }\end{array}$ & 64 kbps \\
\hline 3G 2000 & $\begin{array}{l}\text { - roaming services (voice and data) } \\
\text { - Internet, videocalls } \\
\text { - W-CDMA, ULTRA, GPRS }\end{array}$ & 2 Mbps \\
\hline 4G 2010 & $\begin{array}{l}\text { - mobile TV with HDTV } \\
\text { - Multimedia streaming, videoconferences } \\
\text { - LTE, IP, TDD }\end{array}$ & 100 Mbps \\
\hline 56 & 2020 & $\begin{array}{l}\text { - connectivity regardless of geographical region } \\
\text { - delays in 1ms } \\
\text { - mmWave, MIMO, beamforming }\end{array}$ & 1 Gbps \\
\hline
\end{tabular}

Fig. 1 History and development of mobile technologies

The 4G network, which is currently deployed (year 2020), uses LTE technology, no longer meets the 
requirements of users as well as operators for network transmission speeds, area coverage or network response. The aim of this network is to provide quality and uninterrupted services anywhere and at any time, which, however, is not entirely possible due to the fact that for the functionality of the $4 \mathrm{G}$ network it is necessary to have a solid and stable infrastructure [5]. This does not allow coverage in areas with difficult to access terrain, where it is not possible to build this infrastructure. In the future, a sharp increase in the number of devices requiring an Internet connection is expected, not only other mobile phones or tablets, but also autonomous vehicles, drones or robots, and in this case $4 \mathrm{G}$ networks may no longer be sufficient to ensure connectivity. The current $4 \mathrm{G}$ network is expected to hit its capacity limits in the near future, which would mean unstable connections, lower baud rates, increased latency, or outages due to high terminal growth. The next generation of networks should solve these problems.

Fifth generation networks promise a high increase in transmission speed, which will be around 1 Gbps, a network delay of up to 1 millisecond and simultaneous connection for thousands of devices. For the functionality of today's mobile 4G network, it was necessary to build a fixed infrastructure, which is not possible in some places, so coverage of hard-to-reach areas is almost impossible. 5G networks solve this problem, but it will require the use of technologies such as millimeter waves or the use of a multiantenna system. These technologies will also support the development of other new technologies, such as virtual reality, autonomous vehicles and autonomous drones.

\subsection{Heterogeneous networks}

The development of mobile networks is growing unstoppably. From the first generation of mobile networks, when simple mobile phones were used to make basic voice phone calls, to today's fourth and fifth generation networks, when smartphones with mobile internet are already in use. Over time, people around the world began to use their phones more and more, and this widespread use and demand for wireless communication has significantly contributed to the growth of network traffic. Compared to previous generations of mobile networks, which focused mainly on human-to-human or human-to-content communication, fifth-generation networks will also support human-to-machine or machine-to-machine (M2M) communication itself [6].

One of the main differences between $5 \mathrm{G}$ networks and previous generations is network heterogeneity. In a heterogeneous network, the various components of the network must work together to meet the requirements of users or operators, such as the quality of the services provided, greater network coverage or the use of different spectrums, whether licensed or unlicensed. At the same time, in the area of computing capacity or computing power, devices also support advanced cognitive learning and intelligent resource and network management. Therefore, instead of a simple homogeneous network, a heterogeneous network (HetNet) has become key, which will be an important technology for the functionality of the $5 \mathrm{G}$ network. Heterogeneous networks should integrate several networks intelligently, efficiently and quickly, and ensure their interoperability, such as radio access network (RAN) or Wi-Fi, using different radio access technologies, thus allowing virtualization of individual network components and spectrum resources for maximizing requirements. It will use different carrier frequencies for different approaches to achieve the best quality (QoS), while achieving spectral, cost-effective and efficient connectivity anytime, anywhere and for any device.

In a heterogeneous network, different network elements cooperate on different carrier frequencies, such as:

- Macrocell base stations - have a large coverage area, and there may be several Wi-Fi access points or small cell base stations in the coverage area of one base station,

- Small cell base stations - may use the same spectrum as the macrocells or may use unlicensed and millimeterwave bands,

- Wi-Fi - is an effective supplementary network that allows you to reduce the load on cellular transmission and relieve the congestion of the cellular network.

\subsection{Cognitive radio network}

With advances in research, $5 \mathrm{G}$ networks are expected to provide much higher data traffic, more coverage, low latency, and much higher capacity than current networks. To achieve these goals, new technologies and enhancements need to be integrated into existing $3 \mathrm{G}$ or $4 \mathrm{G}$ network architectures and systems to ensure compatibility between systems. Today's cellular networks use expensive licensed bands and face a problem where, on the one hand, there is a lack of spectrum due to growing demand and, on the other hand, there is insufficient use of spectrum. In a $5 \mathrm{G}$ network, the use of cognitive radio is proposed for dynamic spectrum sharing between devices in the network, which means that the device will have access to both types of spectrum that it will use dynamically. The aim is to increase overall spectrum efficiency and uniform loading [7].

Cognitive radio technology is a major technology in radio technology that has opened up new possibilities for improving the use of congested radio spectrum. Cognitive radio was designed by $\mathrm{J}$. Milota, which is based on software-defined radio and is capable of selfreconfiguration using cognitive intelligence while adapting to the communication process. The incorporation of this technology should solve the problem of inefficient use of spectrum in the $5 \mathrm{G}$ network.

The most important feature of cognitive radio is its cognitive ability, which allows radio technology to monitor information from its surroundings that can identify unused or idle parts of the spectrum at a particular time or location, while providing dynamic access to licensed and unlicensed spectrum. Cognitive radio thus integrates and delivers information to the wireless communication system. Collects spectrum source information, which is then used to optimize spectrum sharing between network devices. Spectrum sharing between devices on a network effectively improves spectrum utilization. Spectrum sharing is essentially the use of the same spectrum by multiple users in terms of time, frequency and location. Spectrum sharing is one of the basic techniques of cognitive radio networks for providing access channels and sharing resources without changing the existing way of spectrum allocation. The spectrum itself is divided into two basic parts for users, 
namely licensed and unlicensed spectrum. Access to individual spectra is based on a hierarchy, where the licensed user is the primary user and the unlicensed is the secondary user. Access to the licensed band, which is shared between licensed primary users and unlicensed secondary users, is restricted to licensed users. In principle, a hierarchical approach is the possibility that the secondary user has access to the spectrum, provided that the licensed user is not currently using the spectrum, i.e. the spectrum is uninterrupted. Otherwise, the secondary user can share the spectrum source with the primary user at the same time, keeping the interference below the threshold. Interferenceresistant cognitive radios can achieve better spectrum utilization and better efficiency by occasionally sharing radio spectrum resources with licensed primary users.

\section{UNMANNED AERIAL VEHICLES}

Unmanned aerial vehicles (UAVs, commonly known as a drones) have found widespread use today. Thanks to high mobility and low costs, they can be used in several areas. They found the most important use mainly in the army, where the deployment of UAVs in conflict areas was applied in order to avoid possible loss of life of pilots. Thanks to the constant reduction of costs, they have also been used in the commercial and civil spheres. Police, firefighters or rescuers can use them to monitor and control traffic or search for forest fires. They can provide early warning of impending natural disasters and assist in rescue operations when the communication network is down due to destruction. Ordinary people use them for recreational purposes or sports. They have also been used in the field of IT, where they can be used to transfer information or to cover hard-to-reach places where it is not possible to build a solid infrastructure. Drones can also provide wireless connections between two or more devices over a long distance, where the individual devices are not within their radio range. An example of user equipment communication and drones is shown in Fig. 2.

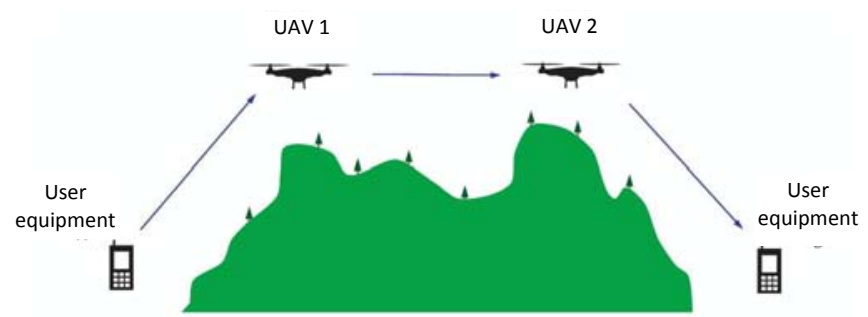

Fig. 2 Communication of terminals with the help of UAV

In terms of application, it is necessary to consider what type of UAV will be used. UAVs are divided into two basic categories. The first category is fixed-wing UAVs, whose main advantage is high speed, but are forced to maintain a continuous forward or backward movement to keep them in the air. Therefore, they are not suitable for use in areas where they need to be used in a stationary position. The second group are UAVs with rotating wings, which are able to move in different directions and the ability to maintain a position in the air at one point, making this type of drone with rotating wings more suitable for deployment in the desired area to provide wireless connection. Thanks to their mobility and easy handling, they also make it possible to cover areas where it is not possible to build a solid infrastructure. These are, for example, places with difficult to access and rugged terrain, or areas that have been affected by natural cadastre, such as earthquakes or tidal waves. These result in the destruction of the infrastructure, ie the base stations, and thus wireless communication is impossible. UAVs are more cost-effective and can be deployed much faster in areas affected by natural disasters. In case of destruction, resp. damage to the communication network, drones can be used effectively, as mobile base stations and their correct location can achieve effective coverage of the affected area, and thus ensure communication, as shown in Figure 3. They can also transport medical supplies to people who will need them. because it will not be possible to deliver it by standard road. They fly at low altitudes, which allows the creation of short-distance communication (LoS). This results in a significant improvement in performance. Their good controllability and fast transferability offer an increase in performance by dynamically adjusting the position of the drones so that the entire required area is covered as effectively as possible. Deployed UAVs can also help existing infrastructure. After partial damage or complete decommissioning of infrastructure due to natural disasters, where base stations are not operational, UAVs are a suitable solution to provide wireless coverage. Thanks to these advantages, wireless communication with UAV support is a promising and integral part of future wireless systems.

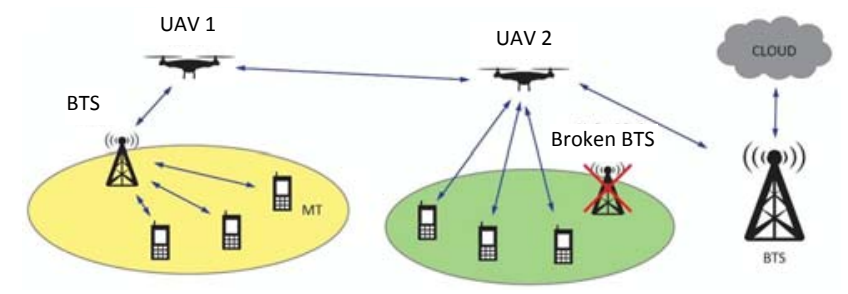

Fig. 3 UAV usage instead of non-functional BTS

\subsection{UAV communication channel}

The initial use of UAVs as mobile base stations were characterized as only one large UAV deployment per task. In this case, the communication network was created based on one large UAV and a number of devices that formed the communication network. Nowadays, the smart applications involve the use of several UAV systems. In a system with multiple UAVs, these are smaller, cheaper and they work in a coordinated manner, making them an effective way to ensure communication in any case of failure.

Thanks to their high mobility, they can be configured to provide services cooperatively and ensure coverage of the area as efficiently as possible. The dynamic behaviour of the network means that UAVs may encounter some problems during long operation, such as a malfunction or low battery. If the UAV is taken out of service, it must be replaced. In some cases, UAVs may be omitted due to energy savings, and therefore their redistribution needs to be automatically reconfigured. In a system with multiple UAVs, removing one UAV from the communication process causes the network to reconfigure itself and maintain communication through other UAVs in the network. This would not be possible in a single large UAV 
system and a UAV outage would cause an absolute network collapse.

The signal coverage of one UAV can continuously change and it depends on the throughput of the communication channel to a user. The communication channel throughput is determined, for example, by the distance between the UAV and the user, the transmitting power of the UAV and the receiving power of the user, interference caused by individual UAVs or also by the number and size of obstacles in the environment, which may reduce its radio range. UAVs are very intelligent in this and are able to work with this information about the given environment and, based on that, they can increase or decrease their transmission power. In the case of large obstacles in the vicinity, such as tall buildings, the radio range of the UAV is limited. The UAV can subsequently reduce the transmission power, which can significantly save energy and thus extend the flight time. Also, by adjusting the altitude at which the UAVs are located, their radio range can be regulated, and thus interference between them can be reduced.

\section{OPTIMIZATION ALGORITHMS OF AUTONOMOUS DRONES LOCATION}

Autonomous drones, due to their excellent mobility and low operating costs, are an effective method for providing services in wireless communication systems. Base stations can be mounted on the UAV and deployed in the field to provide wireless connectivity in areas where no infrastructure is built, such as hard-to-reach areas with rugged terrain or disaster-stricken areas. Unlike ground base stations or base stations mounted on vehicles, autonomous drones such as mobile base stations (MBS) can be deployed in any environment. They can move on any air trajectory based only on the basis of their flight restrictions to provide coverage for terminals in a certain area.

To facilitate the determination of the optimal location of the MBS, it is assumed that the MBSs fly at the same altitude $H$, the positions of the users in 2D space are known and all MBSs have the same radius of coverage $r$ (Fig. 4).

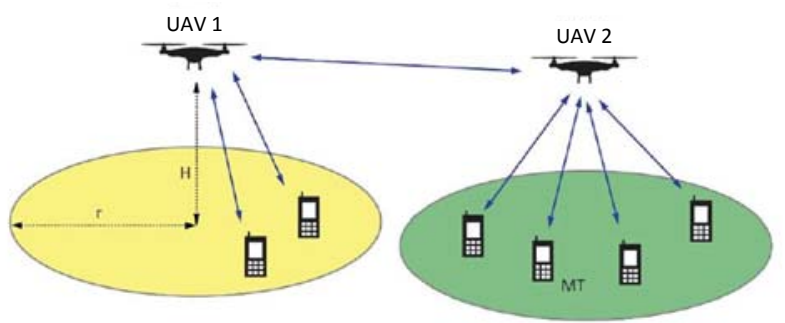

Fig. 4 Coverage of mobile terminals using MBS

No ground base stations are included in the solution. MBS location optimization algorithms focus on deploying the minimum number of MBS required to cover a given area so that each mobile terminal is covered by at least one MBS, but does not exclude that it may be covered by multiple MBS. In case the mobile terminal will be covered by two or more MBS, it is necessary to solve the problem of intercellular interference, for example by correct channel assignment, power management of individual MBS and thus to regulate their range.

Finding the optimal solution for locating the MBS in a given area, and thus ensuring communication for all users in the area, involves the problem of geometric coverage by disks (GDC) of the area, where disk means radio range with radius $r$. The goal of geometric disk coverage is to cover a number of terrestrial terminals with minimal MBS used and minimal interference. To address this issue, several algorithms for optimizing MBS placement will be compared.

\subsection{MBS spiral placement algorithm}

The spiral placement algorithm consists of sequentially positioning the MBSs along the perimeter of a selected area, designed to provide coverage for all users within this area [8]. The perimeter of the area is formed by peripheral users located along the investigation area, which was obtained from the ConvexHull module [9]. Once the marginal users are found, the MBSs are positioned sequentially counterclockwise so that the area gradually shrinks until all users within it are covered. The LocalCover function is used to find the optimal position for MBS to cover as many users as possible.

\subsubsection{ConvexHull library}

Search and assembly of convex hulls, resp. edge points in the area is the basic operation for computational geometry. The ConvexHull library was used to find marginal users in the investigated area [9]. Convex hull in geometry means a co-linear area district or envelope. It defines a closed region using elements from the set that the area contains. The ConvexHull library determines the envelope of all users located in a given area, which is defined as the path connecting edge users along the perimeter. It aligns them counterclockwise with the user at the beginning, located in the lower left corner. There is a hierarchical structure between users in the area. The hierarchical structure determines for which user's priority coverage should be provided. Users acquired through the ConvexHull library are identified as priority users and have a higher coverage priority to ensure that the newly located MBS covers these marginal users in the first place as much as possible.

\subsubsection{LocalCover operation}

The LocalCover task is to find and effectively adjust the position of the located MBS, so as many users as possible to be covered, while constantly supervising the priority of coverage and the hierarchy of individual users located in the area. The function initially works with a set of marginal (primary) users. Users are sorted counter clockwise with the start located in the lower left corner. The first MBS is placed in the position of the first user from the set of selected peripheral users. Subsequently, all other peripheral users within the range of $2 \mathrm{r}$ are searched, which also need to be primarily covered (Fig. 5). In this case, marginal users 2 and 16 are in range $2 r$. The function calculates the optimal position of the MBS so that each primary user in range $2 r$ is covered and the distance between the MBS and the individual primary users is approximately the same. The optimal position for covering found marginal users is shown in Fig. 5 a). Once the optimal position of the MBS is determined so that there are as many primary edge users within radio range as possible, in the next step the MBS 
searches for secondary users who can also be covered and then determines the most optimal position (Fig. 5 b)). This will ensure that as many users as possible are covered.

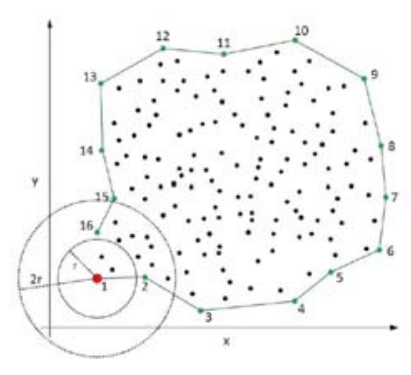

a)

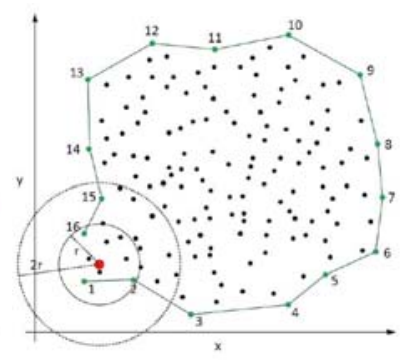

b)
Fig. 5 MBS placement optimization to cover users

After covering all marginal users and all possible secondary users in the total, you must reuse the ConvexHull library and use the LocalCover feature again on the remaining, uncovered users until coverage is provided for all users in the area. Not all covered users, both primary and secondary, are considered in the future of MBS placement and optimization. The set of new fringe users is made up of remaining users who have not yet been covered in the previous steps. These fringe users are also arranged counterclockwise, and then LocalCover is reapplied to find the optimal MBS location and coverage for the remaining users in the area. The location of additional MBSs and the final coverage of all users is shown in Fig. 6 a) b).

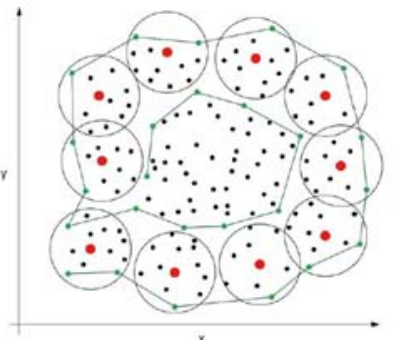

a)

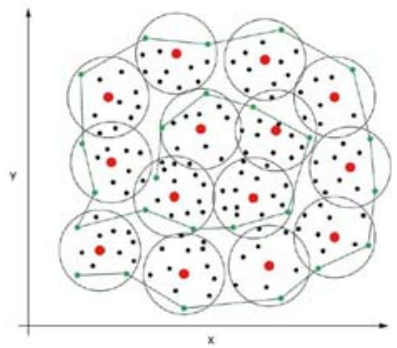

b)
Fig. 6 The final distribution of MBSs and cover all users

\subsection{MBS random placement algorithm}

The MBS random placement algorithm is one of the simplest algorithms. Two models of random distribution are considered. The first is the completely random MBS placement algorithm. The algorithm does not take into account any positions of distributed users in the area. Automatically generates MBS positions until all users in the area are covered for coverage.

The second is a random MBS placement algorithm that already requires fewer MBSs needed to ensure coverage of a given area. In the first step, the algorithm randomly selects a user from all users in the area and places the MBS in the position of the selected user. It then determines for which users in the area it already provides coverage and these users will be deleted from the total set. Deleted users no longer appear in the next run of the algorithm, and the algorithm selects another user from the total set.

\subsection{Strip coverage algorithm}

The general algorithm of strip coverage consists in dividing the plane into several separate strips and solving the location of the MBS in each strip separately. The algorithm itself consists of two steps: 1 . dividing the area into equally wide parts and 2. solving the location and optimization in each strip separately.

There are two different alternatives to the general band coverage algorithm, which differ in the local solution of the MBS location in a given band. The first alternative is to cover the strip with rectangles inscribed in a circle (SCR), depending on the size of the strip and the radius of the MBS coverage. The second alternative is to cover the strips directly by placing an MBS (SCD).

These algorithms are efficient, but there is a problem of interference between the located MBSs in the individual bands. The algorithm covers the strips separately, so only users located in the given strip are at the input of the algorithm. This means that the coverage of users is realized in individual strips separately and the algorithm solves the placement of MBS in a given strip independently of other strips, resp. does not take into account users in other strips. Therefore, a situation may arise where the MBS located in the first strip may inadvertently cover the user in the secondary strip. This inadvertently covered user, located in the second strip, is still referred as uncovered, and the algorithm in solving the placement of the MBS in the second strip will try to cover it again. The advanced band algorithm also addresses the provision of user coverage and the placement of MBS in each band separately, but seeks to avoid this double coverage of one user from two MBS simultaneously. A user who is inadvertently covered by an MBS located in an adjacent, resp. the previous tape, the algorithm considers this unintentionally covered user to be covered and will no longer be taken into account in the solution of covering uncovered users in the tape in which this unintentionally covered user is located. This decision seeks to prevent interference between MBS located in individual bands.

\section{SIMULATIONS EXPERIMENTAL RESULTS}

All algorithms designed to calculate the location of the MBS, to ensure coverage of all users located in the 2D plane, were programmed in the Python programming language. Several algorithms (described above in section 4.) were compared within the number of MBS required to provide wireless coverage to users and the within the time required to calculate the location of MBS in the area.

Several parameters were considered for the simulations, in which the input parameters gradually changed. The size of the area was constant for all scenarios, but the number of users located in the area was changed, resp. points that represent the positions of users in the $2 \mathrm{D}$ plane. Two different scenarios were also considered, in which the reach of one MBS was different. In real conditions, the range of the MBSs is determined by the throughput of the communication channel, which is determined, for example, by the distance between the MBS and the user, the transmitting power of the MBS and the receiving power of the user, and also interference. Therefore, two different scenarios were considered in this view. In the first scenario, 
the radio range of the MBS was assumed to be 1 kilometer, the second scenario was a bit more optimistic, where one MBS had a range of 2 kilometers. For simplicity of MBS location calculations, a uniform altitude is assumed at which all MBSs are located, all have a uniform radio coverage, and reliable communication is ensured. In the experiments for both scenarios we assumed an area with size of $10 \times 10 \mathrm{~km}$ and number of the users was 100 .

After the consecutive application of the above MBS distribution algorithms, it can be concluded that the most optimal MBS placement, in terms of the number of MBS needed to provide coverage, is achieved with the help of the spiral MBS placement algorithm. In this case, the MBSs are located at a relatively good distance from each other, which does not interfere between them, and only $14 \mathrm{MBSs}$ were needed for all users.

The strip coverage algorithm deploys 19 MBSs, which is a less efficient way in terms of the necessary funds spent than the solution achieved with the help of the spiral algorithm. There are more MBSs in the area and they are closer together, which can cause interference between them. With less modification to the extended band coverage algorithm, fewer MBSs have been achieved to provide area coverage. In this case, $17 \mathrm{MBSs}$ were required. The extension of the algorithm in this modifies the standard algorithm by deciding when a user located in an adjacent band is inadvertently covered with an MBS located in the first band, this user will no longer be in the set of users for which coverage needs to be provided. From the above graphical MBS layouts, it can be seen that the extended band algorithm needed a lower number of required MBS to cover all users located in the area.

In a completely random MBS placement algorithm, the algorithm randomly generates MBS positions until coverage is provided for all users in the area. A completely random MBS placement algorithm deploys in average around $151 \mathrm{MBSs}$.

\subsection{Simulation results}

Several scenarios were tested to compare algorithms. The size of the area was constant $(10 \times 10 \mathrm{~km})$ for all scenarios, the number of users in the area (50 to 400 users with a step of 50) and the range of coverage of one MBS (1-2 km) in individual simulations changed. All deployed MBSs have a uniform coverage range in one simulation and all fly at the same altitude. It is ensured that each user located in the area will be covered by at least one MBS, but it is not excluded that it will be within the range of several MBSs.

From the achieved results presented in the table 1, it can be stated that in terms of the need for the number of deployed MBS, the spiral placement algorithm provides the solution with the lowest number of required MBSs. The lower number of deployed MBSs has a positive effect not only on the financial side of the implementation, but also on reducing the chances of possible interference between individual MBSs in the area. The strip coverage algorithm and the extended strip coverage algorithm performed weaker in terms of the number of MBSs required than the spiral placement algorithm. From this point of view, the random MBSs placement algorithm also achieved surprisingly positive results, where there was not such a

\begin{tabular}{cccccc}
\hline $\begin{array}{c}\text { No. of } \\
\text { users }\end{array}$ & Spiral & $\begin{array}{c}\text { Strip } \\
\text { base }\end{array}$ & $\begin{array}{c}\text { Strip } \\
\text { extended }\end{array}$ & Random & $\begin{array}{c}\text { Totally } \\
\text { Random }\end{array}$ \\
\hline \hline 50 & $33 / 18$ & $34 / 18$ & $34 / 18$ & $34 / 20$ & $631 / 115$ \\
\hline 100 & $52 / 21$ & $59 / 23$ & $58 / 23$ & $54 / 24$ & $665 / 183$ \\
\hline 150 & $60 / 28$ & $71 / 30$ & $66 / 30$ & $62 / 33$ & $703 / 351$ \\
\hline 200 & $68 / 31$ & $70 / 36$ & $70 / 33$ & $75 / 38$ & $860 / 209$ \\
\hline 250 & $79 / 34$ & $81 / 36$ & $80 / 35$ & $87 / 39$ & $960 / 249$ \\
\hline 300 & $81 / 34$ & $87 / 40$ & $85 / 40$ & $88 / 42$ & $998 / 266$ \\
\hline 350 & $90 / 35$ & $101 / 38$ & $99 / 37$ & $105 / 39$ & $1105 / 291$ \\
\hline 400 & $96 / 41$ & $105 / 48$ & $103 / 44$ & $106 / 47$ & $1210 / 327$ \\
\hline
\end{tabular}

Tab. 1 Number of MBSs required to ensure user coverage within an MBS range $(1 \mathrm{~km} / 2 \mathrm{~km})$

large difference in the number of MBSs deployments required to provide coverage. However, in this case, when the MBSs are placed randomly, more interference may occur between the MBSs compared to other algorithms.

In terms of the time required to calculate the location of the MBS in the area, it is possible to state from the achieved results shown in the tables that the best results are achieved by the band coverage algorithm. This is several times less time required to calculate the location compared to other algorithms, which can have a great advantage in timelimited situations. From this point of view, the extended tape coverage algorithm is slightly worse than the original tape coverage algorithm, on the other hand, in this case, the number of MBS required to ensure coverage of all users in the area is reduced. The worst results in both cases were achieved by a completely random placement algorithm.

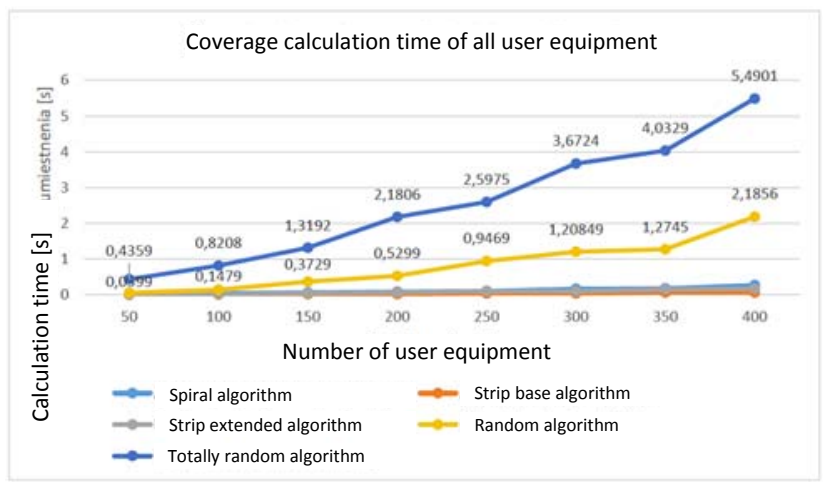

Fig. 7 Comparison of computation times for MBSs placement

From the achieved results of the algorithms listed in Fig. 7, it can be stated that in terms of the time required to calculate the location of the MBS, the best results are achieved by the band coverage algorithm. Although this algorithm ranks in the number of MBSs required to ensure third-party user coverage in the area, its speed is significantly higher compared to other algorithms. According to the measured values, the band coverage algorithm needs about half the time to cover all users in the area than the extended band algorithm and about three times the time compared to the spiral placement algorithm. The worst results in both cases were achieved by a totally random MBS placement algorithm. This algorithm needed 
many times higher computational time to calculate the location of MBS, higher number of MBS and their placement was chaotic. Therefore, it is very important to prioritize the use of the researched algorithms. The results of the simulations clearly demonstrated their advantages and disadvantages.

\section{CONCLUSIONS}

The aim of this work was to introduce and implement algorithms for MBSs position optimization. The time required to calculate the location and to optimize the location of autonomous drones, such as mobile base stations, and the number of MBS required to provide wireless coverage of the area were compared. It can be seen from the measured results that each algorithm has its advantages and disadvantages. One algorithm can find a more efficient MBSs distribution in the area, but at the cost of the higher computational time, another one can find an easier MBSs deployment in a very fast time, but with a higher number of MBSs.

From this point of view, it is necessary to determine in advance which claims will be primarily emphasized, resp. for what purpose it will be necessary to deploy MBSs. The spiral placement algorithm was able to provide coverage for all users in the area with the lowest number of MBSs required, which has great financial benefits and also relatively avoids interference problems. On the other hand, the strip algorithm needs them a little more to implement the coverage, but the time required to calculate the MBS location is much faster than the other algorithms. The advanced band algorithm modifies the standard band algorithm to decide when a user may be inadvertently covered by an MBS located in the previous band and at the same time by an MBS located in the band in which the user is located. If the user is inadvertently covered with MBS from the previous strip, it will no longer be taken into account in the further course of the algorithm. Therefore, the extended band algorithm becomes the middle ground between the spiral algorithm and the standard band algorithm. It significantly reduces the computation time required by the spiral algorithm while reducing the number of MBS required to ensure coverage of all users in the area, compared to the standard band algorithm. The MBS randomization algorithm also performed positively, but the computation time was significantly higher. The worst results in both cases were achieved by a completely random MBS placement algorithm.

\section{ACKNOWLEDGMENTS}

This research was supported by the by the Slovak Research and Development Agency, project number APVV-18-0214 and APVV-18-0368, and by the Scientific Grant Agency of the Ministry of Education, Science, Research and Sport of Slovakia under the contract $1 / 0268 / 19$.

\section{REFERENCES}

[1] ZENG, Y. - ZHANG, R. - LIM, T. J.: "Wireless communications with unmanned aerial vehicles: opportunities and challenges," IEEE Commun. Mag., vol. 54, no. 5, pp. 36-42, May 2016.

[2] BOR-YALINIZ, R. I. - EL-KEYI, A. YANIKOMEROGLU, H.: "Efficient 3-D placement of an erial base station in next generation cellular networks," in Proc. IEEE Int. Conf. Commun. (ICC), May 2016, pp. 1-5.

[3] ZENG, Y. - WU, Q. - ZHANG, R.: "Accessing From the Sky: A Tutorial on UAV Communications for $5 \mathrm{G}$ and Beyond," in Proceedings of the IEEE, vol. 107, no. 12, pp. 2327-2375, Dec. 2019.

[4] HE, J. et al.: "Machine Learning based Network Planning in Drone Aided Emergency Communications," 2020 IEEE 91st Vehicular Technology Conference (VTC2020-Spring), Antwerp, Belgium, 2020, pp. 1-5.

[5] CABREIRA, T. M. - BRISOLARA, L. B. FERREIRA JR., P. R.: Survey on Coverage Path Planning with Unmanned Aerial Vehicles. Drones 2019, 3, 4 .

[6] CHEN, B. - RHO, S.: "Autonomous Tactical Deployment of the UAV Array Using SelfOrganizing Swarm Intelligence," in IEEE Consumer Electronics Magazine, vol. 9, no. 2, pp. 52-56, 2020.

[7] LYU, J. - ZENG, Y. - ZHANG, R.: "Cyclical Multiple Access in UAV-Aided Communications: A Throughput-Delay Tradeoff," IEEE Wireless Commun.Lett., to appear, 2016.

[8] LYU, J. - ZENG, Y. - ZHANG, R. - LIM, T. J.: "Placement Optimization of UAV-Mounted Mobile Base Stations," in IEEE Communications Letters, vol. 21, no. 3, pp. 604-607, March 2017.

[9] DUCKHAM, M. - KULIK, L. - WORBOYS, M. GALTON, A.: "Efficient generation of simple polygons for characterizing the shape of a set of points in the plane," Pattern Recognition, vol. 41, no. 10, 2008.

Received December 12, 2020, accepted January 11, 2021

\section{BIOGRAPHIES}

Adrián Stosil is the student at the Department of Electronics and Multimedia Communications, Faculty of Electrical Engineering and Informatics, Technical University of Košice. His research interests include data analysis and modelling network infrastructures, theory and simulation of complex systems.

Marcel Vološin is the PhD. student at the Department of Computers and Informatics, Faculty of Electrical Engineering and Informatics, Technical University of Košice. His research interests include real-time spectrum trading schemes, modelling of network complexity and real time mobility. 
Taras Maksymyuk (M'14) is a Research Professor with the Telecommunications Department, Lviv Polytechnic National University, Lviv, Ukraine. He serves as an Associate Editor of the IEEE Communications Magazine.
Gabriel Bugár is a Research Assistant at the Department of Electronics and Multimedia Communications, Faculty of Electrical Engineering and Informatics, Technical University of Kosice, Slovakia. His research interests include cognitive networks, image processing, security and e-learning. 